\title{
ATOMISTIC DYNAMICS OF THE RICHTMYER-MESHKOV INSTABILITY IN CYLINDRICAL AND PLANAR GEOMETRIES
}

\author{
V. V. Zhakhovskii ${ }^{1}$, S. V. Zybin ${ }^{2}$, S. I. Abarzhi ${ }^{3}$, and K. Nishihara ${ }^{1}$ \\ ${ }^{1}$ Institute of Laser Engineering, Osaka University, Osaka, 565-0871, Japan \\ ${ }^{2}$ Materials and Process Simulation Center, California Institute of Technology, Pasadena, CA 91125, USA \\ ${ }^{3}$ Center for Turbulence Research, Stanford, CA 94305, FLASH Center, The University of Chicago, IL 60637
}

\begin{abstract}
We apply molecular dynamics (MD) simulations to study the evolution of the shock-driven Richtmyer-Meshkov instability (RMI) in the cylindrical and planar geometries. Compared to traditional hydrodynamic simulations, MD has a number of fundamental advantages: it accounts for strong gradients of the pressure and temperature, and captures accurately the heat and mass transfers at the early stage (shock passage) as well as the late stage (perturbation growth) of the instability evolution. MD has no hydrodynamic limitations for spatial resolution and thermodynamic quasiequilibrium at atomic scale. We study the instability evolution for different perturbation modes and analyze the role of the vorticity production for RMI dynamics.
\end{abstract}

Keywords: molecular dynamics, shock wave, hydrodynamic instability, Richtmyer-Meshkov

PACS: $02.70 . \mathrm{Ns}, 47.20 . \mathrm{Bp}, 47.40 . \mathrm{Nm}, 52.57 . \mathrm{Fg}, 62.50 .+\mathrm{p}, 68.35 . \mathrm{Ja}$

\section{INTRODUCTION}

Recent advances in the computing power have created great opportunities for modeling nonequilibrium phenomena via molecular dynamics (MD) simulations [1]. With a few billions of atoms simulated, MD can grasp at atomistic level the essentials of many non-equilibrium processes in shock physics, detonation and hydrodynamics $[2,3]$. Several representative examples are the phase transitions under shock compression, chemical reactions, and laser ablation of ultra-fast heated solids [4]. In laser ablation, a shock wave induced by laser irradiation interacts with the target imperfections and results in the RichtmyerMeshkov instability (RMI) of the ablation front $[5,6]$. The modeling of RMI by means of continuous dynamics simulations is hard problem [7]. It has certain limitations in capturing the mass and heat transfers across the front, especially in the cases of strong shocks and the nonlinear growth of the perturbations [8].
Here we report MD simulations of RMI in curved and planar geometries. Compared to continuous dynamics simulations [7], our method has a number of fundamental advantages [9]. It accounts for strong gradients of pressure and temperature and captures accurately the heat and mass transfers across the front. MD has much less limitations for spatial resolution at atomic scale and thermodynamic equilibrium than the hydrodynamic approach. We perform the computations using with parallel MD code with new load-balancing algorithm [10] capable to redistribute effectively between the processors the highly non-uniform work load, which characterizes the shock wave passage through the materials of different densities. The simulations provide the unmatched insight for capturing the instability dynamics at nano-scales, and highlight the role of the vorticity production for the nonlinear dynamics of RMI.

\section{SIMULATION MODEL}


In our MD simulations, the interaction between the atoms is described by a modified Lennard-Jones (LJ) potential

$$
\begin{aligned}
\phi_{L J}(r) & =4 \varepsilon\left[(\sigma / r)^{12}-(\sigma / r)^{6}\right] \\
& -a_{2}\left(r^{2}-r_{0}^{2}\right)^{2}-a_{3}\left(r^{2}-r_{0}^{2}\right)^{3}
\end{aligned}
$$

where $r$ is the distance between a pair of atoms, $r_{c u t}$ $=2.5 \sigma$ is the short-range cut-off, and $\sigma, \varepsilon$ are the $\mathrm{LJ}$ parameters. The spline term is added in the $\mathrm{LJ}$ potential (1) to get smooth second-order derivatives [1], and the spline coefficients are $\mathrm{a}_{2}=-3.5289 \times 10^{-3}, \mathrm{a}_{3}=5.75868 \times 10^{-4}$. The atomistic mass is $\mathrm{m}_{\mathrm{a}} / 48$. These parameters set the reduced MD units $(m d u)$, which are used hereinafter as basic dimensions. For argon, for instance, the characteristic scales are: the length is $\sigma=3.405 \AA$, the temperature is $\varepsilon / \mathrm{k}_{\mathrm{B}}=119.8 \mathrm{~K}$, the velocity is $1094 \mathrm{~m} / \mathrm{s}$, the density is $1.68 \mathrm{~g} / \mathrm{cm}^{3}$, and the time unit is $\left.\sigma\left(\left(\mathrm{m}_{\mathrm{a}} / 48\right) / \varepsilon\right)\right)^{1 / 2}=3.113 \times 10^{-13}$.

To simulate RMI in cylindrical geometry, we place initially the atoms into a rectangular MD cell inside a cylindrical domain surrounded by a piston as a cylinder wall. The piston is modeled by an external potential $\sim[r-R(\varphi, t)]^{2}$. The position of the piston depends both on angle (if the boundary is perturbed) and on time to generate the shock wave. The total number of atoms involved in the simulations is $\sim 4.2 \times 10^{6}$. The MD cell has the dimensions $L_{x} \times L_{y} \times L_{z}$, where $L_{x}=L_{y}=280 \mathrm{~nm}$, and the cell thickness $L_{z}=74.2 \mathrm{~nm}$. The periodic boundary conditions are imposed along the axis of the cylinder (z-axis).
To keep the initial equilibrium state of the LJ liquid, the Langevin thermostat is used in the preparation stage. The initial radius of the cylinder is fixed, $R_{0}=134.3 \mathrm{~nm}$, all atoms have the same atomic mass $m_{a}=48$, the initial temperature is $T_{0}=0.72 \mathrm{mdu}$, and density number is $n=0.79 \mathrm{mdu}$. For a perturbed boundary, the initial position of the cylindrical piston is $R(\varphi)=R_{0}[1+\delta \sin (m \varphi)]$, where $m$ is the mode number and $\delta=0.05$ is the relative intensity determining the initial amplitude of the perturbation, $\Delta=2 \delta \cdot R_{0}$. At the end of the preparation stage an equilibrium state is achieved with uniform density distribution.

To model the dynamics of two materials with different densities, at the beginning of the simulation stage, we change instantly the atomic masses of 'heavy' particles outside the interface $\left(m_{b}=16 m_{a}\right)$ and their velocities $\left(v_{b}=v_{a} / 4\right)$ to hold the uniform temperature and pressure across the system. Interaction between the atoms is described by the same LJ potential (1) without changes in the other parameters. At $t=0$ the piston starts to move with a constant velocity $u_{\mathrm{p}}$ and at time $t=16$ it is removed from the system. The speed of sound of the light material is $c=0.7 m d u$, and the Mach number is $M=3.3$ for a shock wave generated by a piston with $u_{\mathrm{p}}=1.0 \mathrm{mdu}$. The modeling of the shock passage through the materials of different densities is characterized by a highly non-uniform work load of the processes. To resolve this computational difficulty, we develop a special, very efficient loadbalancing algorithm $\mathbf{M P D}^{3}$ (material particle dynamic domain decomposition) based on the Voronoi decomposition [10].
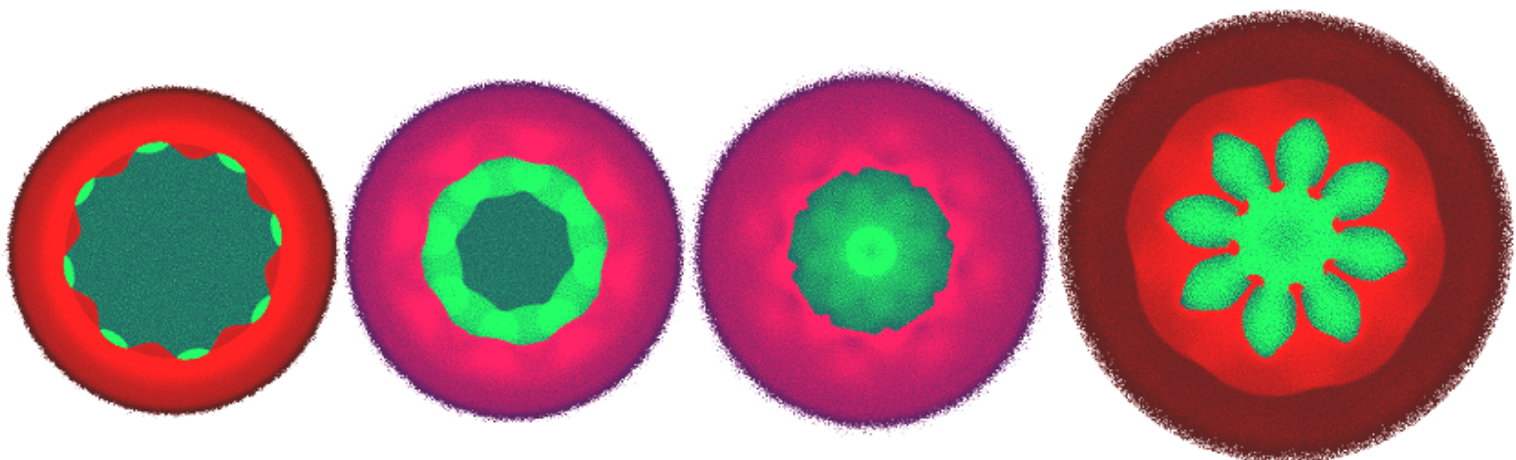

FIGURE 1. MD simulation of the RM instability of heavy-light liquid interface for mode number $m=8$ and piston velocity $u_{\mathrm{p}}=1.0 \mathrm{mdu}$. Brightness corresponds to atom density. (Left, $t=68.3$ ): shock front hits material interface; reflected wave is rarefaction wave. (Left center, $t=75$ ): transmitted SW and Mach stems. (Right center, $t=139.5$ ): spikes and bubbles begin to grow after hitting of the interface by SW reflected from origin. (Right, $t=267.2$ ): mixing zone mounts the asymptotic stage. 
The modeling of RMI evolution in the planar 2D geometry is very similar to that in the cylindrical case, except for the difference in the piston potential, the boundary conditions at the infinity and the initial conditions at the material interface.

\section{RESULTS AND DISCUSSION}

Figure 1 shows the characteristic snapshots of the RM instability of perturbed interface for a converging shock wave (SW), which propagates from the heavy to the light liquid. The velocity fields are presented in Fig. 2. When the shock front

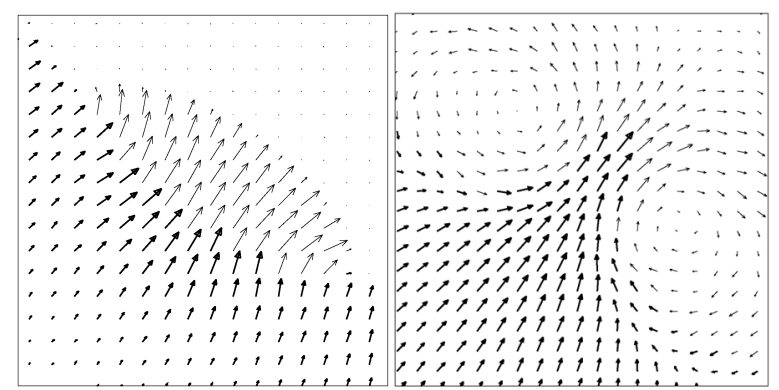

FIGURE 2. Flow velocity map at heavy-light interface in case of the RM instability for $m=8$. Dark arrows correspond to heavy material, light arrows - light material. Corresponding density maps are shown in Fig.1. (Left, $t=68.3$ ): SW-interface interaction. Each arrow is an averaging of 320 atom velocities roughly. Frame size is $70 m d u$. (Right, $t=139.5$ ): flow velocity map around spike. Each arrow represents the mean velocity about 100 atoms. Frame size is $40 \mathrm{mdu}$. hits the interface, the transmitted shock and rarefaction wave are produced and move away from the material interface. The vortices are allocated around the material interface and cause the spike formation (see Fig. 2). Figure 1 shows the development of RMI in the late-time asymptotic regime.

We define the thickness of the mixing zone $\Delta$ as a distance between the maximum and minimum of the interface positions along the radius. For a comparison with the symmetrical case we draw mean position (circles) of the cylindrical interface $m=0$ as a function of time. Emphasize that for $m>>1$, small perturbations are unavoidable, see Figure 1. They are caused by the atomistic fluctuations and grow as artificial ripples. The simulations indicate three distinct key points in RMI evolution. The first point corresponds to a contact of the shock front with a bottom of the ripple. After the contact, the maximum and minimum are closing in time, and the phase reversion occurs. For a higher mode number the reversion happens at an earlier time. At the second distinct time point, the reflected shock reaches and accelerates the interface. At the third distinct key point with $t \sim 180$ the secondary reflected shock pushes the interface outward.

We estimate the asymptotic growth-rate from the differentiation of the asymptotic value of the thickness of the mixing zone $\Delta=\Delta(t)$. Due to the numerical differentiation the growth rate $d \Delta / d t$ is a noisy value. In the case of $m=8$ the perturbation grows with time as $\Delta_{8} \sim t^{0.45}$, for $m=5$ the value of

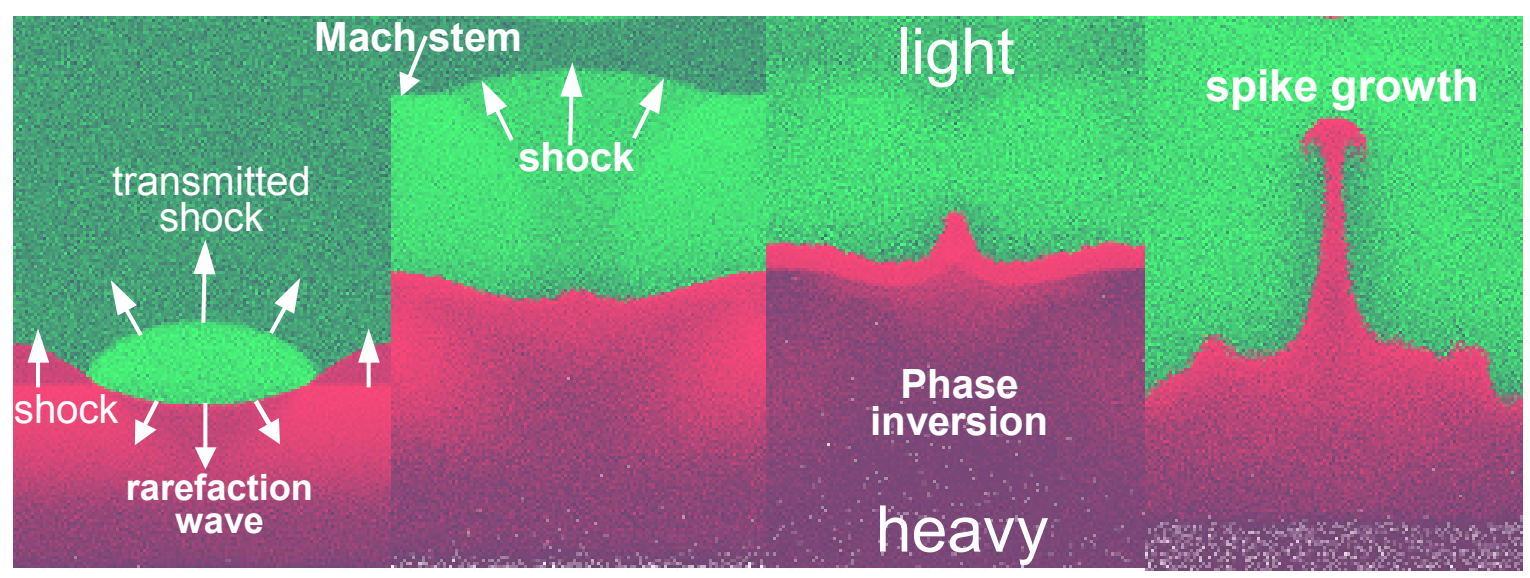

FIGURE 3. Density map at light-heavy liquid interface for the planar RMI with a finite amplitude initial perturbation. 
$\Delta_{5} \sim t^{0.3}$, whereas for $m=3 \Delta_{3} \sim \ln (\mathrm{t})$. Hence the growth rates decay are the following: $d \Delta_{8} / d \sim t^{-0.55}$, $d \Delta_{5} / d \sim t^{-0.7}$, and $d \Delta_{3} / d \sim t^{-1.0}$ respectively.

We apply the MD approach to model the RMI evolution in planar two-dimensional case. Figure 3 shows the development of RMI from the initial to the nonlinear regime induced by a shock propagating from the light to heavy fluid for a finite-amplitude initial perturbation. Many features of RMI dynamics in the planar and cylindrical cases are very similar, such as the formation of the rarefaction and transmitted shock waves, the Mach stems caused by the intersection of the shock waves, vorticity production and the mushroomtyped shape of the spike, etc. (see Figures 1 and 3). However, compared to the cylindrical case, in the planar cases the growth-rate is significantly slower, the transition to the asymptotic regime occurs at a later time, and the formation of bubbles is less pronounced. In the planar cases the amplitude of the shock wave is constant, whereas in cylindrical case the converging shock accelerates. In addition, in cylindrical geometry the shock reflected from the center of the cylinder hits the interface repeatedly and causes significant growth of the instability.

We perform as well MD simulations of RMI in solids [11]. For a strong enough shock, the material melts and the initial growth of the perturbations at the solid-solid interface evolves gradually into the instability with partial (liquid-solid) or complete (liquid-liquid) melting transition of the material at

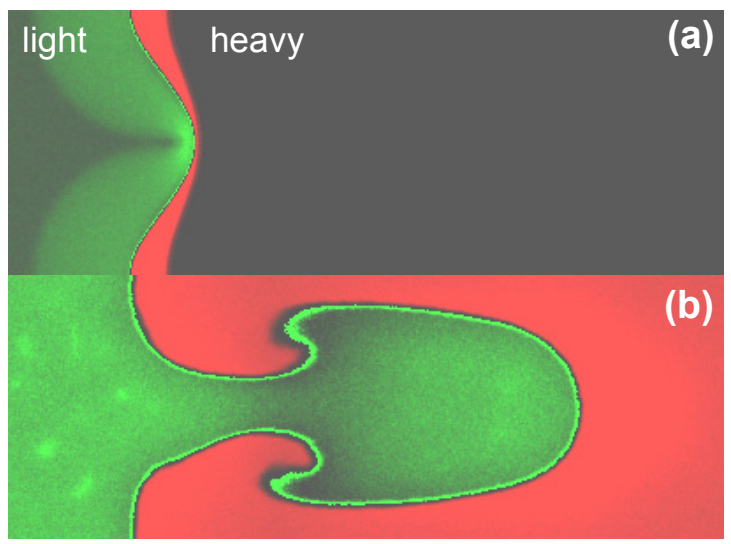

FIGURE 4. Density map at the light-heavy planar interface for the (a) early, and (b) late period of the RM instability in solids with shock-induce melting transition (MD simulations with about 20 million of atoms). the interface. A characteristic snapshot of the late period of RM evolution at the stage of the solid-toliquid transformation in the shock wave is presented in Figure 4. Here, the MD approach provides an unmatched capability to capture the intrinsic details of material flows in a presence of the melting transition.

To summarize, we have performed MD simulation of RMI in cylindrical and planar geometries. We have demonstrated that MD method is able to capture the hydrodynamic instabilities in multiphase material flows in close details at atomistic scales.

\section{ACKNOWLEDGEMENTS}

SVZ is supported by DOE ASC, ONR and ARO MURI. SIA is supported by NRL and by DOE ASC, contract \#B523820.

\section{REFERENCES}

1. Zhakhovskii, V. V., Zybin, S.V., Nishihara, K., and Anisimov, S.I., Phys. Rev. Lett., 83, 1175 (1999).

2. Kadau, K., Germann, T.C., and Lomdahl, P.S., Int. J. Modern Phys. C, 15, 193 (2004).

3. Nakano, A., Kalia, R. K., Nomura, K., Sharma, A., Vashista, P., Shimojo, F., van Duin, A.C.T., Goddard III, W. A., Biswas, R., Srivastava D., submitted to Supercomputing (2005).

4. Anisimov, S.I., Zhakhovskii, V. V., Inogamov, N.A., Nishihara, K., Oparin, A.M., Petrov, Yu. V., JETP Lett., 77(11), 606 (2003).

5. Richtmyer, R. Comm. Pure Appl. Math. 13, 297 (1960).

6. Meshkov, E., Sov. Fluid Dynamics, 4, 101 (1969).

7. Kamm J.R., et al., LANL Report LA-UR-99-2783, (1999).

8. Wouchuk, J.G. and Nishihara, K., Phys. Plasmas. 4, 1028 (1997).

9. Zhakhovskii, V.V., Nishihara, K., and Abe, M., in: Proc. of 2nd Int. Conf. on Inertial Fusion Science and Applications (IFSA2001, September 9-14, 2001 Kyoto, Japan), p.106-109.

10. Zhakhovskii, V. V., et al., in: IEEE Proc. of the 5th Int. Symp. on Cluster Computing and Grid (CCGrid 2005, May 9-12 Cardiff, Wales, UK), Paper ID 63.

11. Zybin, S. V., Zhakhovskii, V. V., Bringa, E.M., Abarzhi, S.I., and Remington, B., these proceedings. 